\title{
Melastoamataceae de tres regiones del Chocó
}

\section{Three regions Melastomateceae Chocó}

\author{
Enrique Rentería Arriaga ${ }^{1}$, Leider Palacios ${ }^{2}$, Jhon J airo Cuesta ${ }^{3}$, Zulmary Valoyes ${ }^{4}$
}

\section{RESUMEN}

La región del Chocó en Colombia es una de las áreas más húmedas del mundo. En el herbario Chocó reposan 24 géneros y 128 especies de los cuales, dos géneros y 53 especies se encuentran en la colección. Existen 87 melastomatáceas indeterminadas y 310 sólo determinadas hasta género. Las regiones fitogeográficas mejor representadas son la selva pluvial central, la región del San Juan, San José del Palmar y la costa Pacífica. Las regiones más pobres son las tierras altas del Carmen de Atrato, la región de Urabá, la serranía del Darién y la selva húmeda del norte. En el presente trabajo se realizaron tres muestreos: en Tutunendo, en Tadó y en Pie de Pató (ciudad Baudó); en cada uno de ellos se analizó 0.4 hectáre a donde se colectaron especímenes al azar y se obtuvieron 87 especies, con los siguientes resultados: 16/41, 19/55 y 13/37 (géneros/ especies), Tutunendo, Tadó y Baudó, respectivamente. Es decir, para un total de 19 géneros y 87 especies, una especie nueva en el géneroTessmannianthus para Colombia, con la posibilidad de que en otros géneros haya nuevas especies, por ejemplo Henrriettella.

Palabras clave: Melastomataceae;Tutunendo; Alto Baudó; Tadó.

\section{ABSTRACT}

The Choco region in Colombia is considered one of the wettest areas of the world, Forero \& Gentry (1989). Lie in the herbarium CHOCO 24 genera and 128 species of which two genera and 53 species rests in the collection. There are 87 and 310 indeterminate Melastomataceae only identified to genus. Phytogeographical regions were better represented, the central rainforest, the region of San Juan, San José del Palmar, costa pacifica, and the poorest, the highlands of Carmen de Atrato, the region of Urabá, the Serrania del Darien and jungle humidity norte. This work were three sampling: Tutunendo, and in Tadó Pie de Pato (city Baudó) in each 0.4 hectare was analyzed, and specimens were collected randomly where was 87 species, with the following results: 16/ 41,19/55 and 13/37 (genus/species, Tutunendo, Tadó and Baudó) respectively. ie, for a total of 19 genera and 87 species, a new species in the genus Tessmannianthus to Colombia, with the possibility that other genres are new species for example Henrriettella.
1. Biólogo, MSc en Sistemática Botánica, Grupo de Investigación en Biosistemática, Universidad Tecnológica del Chocó «Diego Luis Córdoba», Director general de la Fundación Jardín Botánico Jotaudo, Chocó, Colombia.

e-mail: enriquerenteria11@ @otmail.com

2. Biólogo con énfasis en recursos naturales, Grupo de Investigación en Biosistemática, Universidad Tecnológica del Chocó «Diego Luis Córdoba», Chocó, Colombia.e-mail: leipal@hotmail.com

3. Biólogo con énfasis en recursos naturales, Grupo de Investigación en Biosistemática, Universidad Tecnológica del Chocó «Diego Luis Córdoba», Chocó, Colombia.e-mail: jhojacusa@gmail.com

4. Bióloga con énfasis en recursos naturales, Especialista en Administración de Recursos Naturales, Grupo de Investigación en Biosistemática, Universidad Tecnológica del Chocó «Diego Luis Córdoba», Chocó, Colombia. e-mail: zulmaryvaloyes@gmail.com

Keywords: Melastomataceae; Tutunendo; Alto Baudó; Tadó.

\section{INTRODUCCIÓN}

Se estima que en el neotrópico pueden existir 4200-4500 especies de Melastomataceae en 166 géneros (Takhtajan 1997), para Colombia se registran aproximadamente 61 géne- ros (Quiñones 2001).

La familia Melastomataceae Jussieu (1789) pertenece a la división Magnoliophyta, a la clase Magnoliopsida y al orden Myrtales Cronquist (1981).

Los estudios de la familia Melastomataceae en la zona son 
pocos y presentan una carencia de información confiable para la determinación de sus especies, muchas de las cuales muestran una distribución restringida a zonas específicas (endemismos) (Borchsenius 1987). A pesar de ello, esta familia se constituye como un indicador ecológico importante por su ecología y su taxonomía diversificada, que además suministra información extrapolable a otros grupos (patrones de distribución y riqueza).

La familia Melastomataceae (tunos, mayos y siete cueros), se caracteriza por presentar hierbas anuales o perennes, arbustos o árboles pequeños, a veces epifitas o trepadoras; plantas hermafroditas o raramente dioica; hojas simples, opuestas, decusadas generalmente con 3-9 nervios longitudinales que se organizan en la base de la lámina o nerviación plinervia, raramente pinnatinervia; inflorescencia en panícula o cimas terminales o axilares o flores solitarias; fruto una cápsula o una baya; semillas pocas a muchas de varias formas, sin endosperma (Almeda 2001).

El conocimiento sobre la diversidad y distribución de este grupo en el departamento de Chocó es bastante pobre, porque es un grupo de interés para los investigadores, pese a que siempre se informa en todos los estudios florísticos, como una de las familias más representativas. Este trabajo se realizó como un aporte al conocimiento sobre esta familia, a la distribución biogeográfica de sus representantes en el Chocó y para aumentar las colecciones del herbario de la UTCh, que contribuirá al desarrollo del estudio biosistemático de ésta, dentro del marco del estudio de la familia para el Chocó biogeográfico.

Los resultados alcanzados refrendan la importancia de la realización de estos estudios monográficos para el departamento del Chocó.

\section{ÁREA DE ESTUDIO}

El departamento del Chocó tiene una extensión de 47.000 $\mathrm{km}^{2}$ aproximadamente, equivalente a $4 \%$ del total de la extensión del país. Está conformado por 31 municipios, en tres de los cuales (Quibdó, Istmina y Riosucio) se concentra gran parte de la población, para un total de 423.098 habitantes, según cifras estimadas a partir de la población censada en 1993.

De esta población, $90 \%$ es de raza negra; $6 \%$ es mulata y blanca y el $4 \%$ restante es indígena. El departamento está localizado en la región pacífica al noroccidente colombiano entre $\operatorname{los} 4^{\circ} 10^{\prime}$ y $\operatorname{los} 8^{\circ} 10^{\prime}$ de latitud norte y entre $\operatorname{los} 76^{\circ}$ y $78^{\circ}$ de longitud oeste, es la única región de Sur América que posee costas en los dos océanos.

Los ríos y las áreas de ciénagas influyen en el clima húmedo que prevalece, así como su zona que tiene una altísima precipitación pluvial y es reconocida como una de las más húmedas del planeta. El régimen de lluvias es permanente, registrándose hasta $11.770 \mathrm{~mm}$ para varios años en Tutunendo; estos datos explican por qué esta región es el lugar más húmedo del mundo (Sota 1972). De acuerdo con Buston y Ramírez (1977), el área más lluviosa se localiza en La Vuelta, al sur de Quibdó, con un promedio de $9.100 \mathrm{~mm}$ después de 31 años de datos directos. Estas condiciones de ubicación y clima, son la base del hábitat de la más variada y rica gama de recursos de flora y fauna, construyendo una importante reserva patrimonial, que alberga dentro de sus límites territoriales, el Parque Nacional Ensenada de Utría, el Parque de las Orquídeas en los límites con el departamento de Antioquia, el Parque Nacional Natural de Tatamá, mientras que en la frontera con Panamá se ubica el Parque Nacional de los Katíos (Plan de Desarrollo 2004-2007).

Limita al norte con la República de Panamá y el mar Caribe, al oriente con los departamentos de Antioquia, Risaralda y Valle del Cauca, al sur con el Valle del Cauca y al occidente con el océano Pacífico.

En el Chocó se distinguen cinco unidades fisiográficas: planicie marina, formas aluviales, colinas, serranías y cordilleras, todas con representación de Melastomataceae que crecen en nuestro departamento. En el mapa ecológico de Colombia (IGAC 1977; citado por Forero y Gentry 1989), presenta ocho zonas de vidas: bosque muy húmedo tropical (bmh-T), bosque muy húmedo premontano (bmh-PM), bosque húmedo tropical (bh-T), bosque pluvial montano bajo (bp-MB), bosque muy húmedo montano bajo (bmh-MB) y bosque pluvial montano(bp-m).

Fisiográficamente, Forero y Gentry 1989, reconocen ocho regiones fitogeográficas:

Región de Urabá. Ubicada al norte, en el bajo río Atrato, en la costa chocoana sobre el mar caribe, está cubierta en su mayor parte por bosque húmedo tropical (bh-T); es la única con estación seca marcada.

Serranía del Darién. Las tierras altas a lo largo de la frontera con Panamá, que alcanzan los 1540 m en el Alto del Nique y los $1900 \mathrm{~m}$ en la cumbre del cerro Tacarcuna.

Selva húmeda del norte. Se localiza hacia el sur de la región de Urabá, se extiende desde los límites con Antioquia hasta la serranía del Darién, al norte de Riosucio, conformada en su mayor parte por bosque muy húmedo tropical, según Holdridge.

Región de la costa Pacífica. Abarca la planicie costera del Pacífico desde la frontera con Panamá hasta la desembocadura del río San Juan, incluye el Alto del Buey que alcanza una altura de $1200 \mathrm{~m}$ (la tercera salida se realizó a la zona del Alto Baudó, Ciudad Baudó o Pie de Pató)

Tierras altas del Carmen de Atrato. Es la región del alto río Atrato, en la vertiente occidental de la cordillera Occidental por encima de los $1.000 \mathrm{~m}$.

Selva pluvial central. Localizada en el valle del río Atrato, en los alrededores de Quibdó, donde se localiza la mayor 
pluviosidad del Departamento; la selva pluvial tropical de esta zona es una de las más ricas del mundo en número de especies (la primera salida se realizó a Tutunendo)

Región de San José del Palmar. En las cercanías de la localidad con el mismo nombre, en el suroriente del Departamento y en las estribaciones de la cordillera Occidental. Esta región incluye el Cerro del Torrá que alcanza los $2.700 \mathrm{~m}$ de altura.

Región del río San Juan. Incluye el valle del río San Juan, desde Istmina hacia el sur. La mayor parte de esta región es de transición entre el bosque pluvial y el bosque muy húmedo tropical, con 6000-7500 mm de precipitación anual. Nuestra segunda salida se concentró en esta región, es decir, en la estación que posee el IIAP (Alto San Juan, Tadó entre Angostura y Playa de Oro).

\section{MÉTODOS}

El trabajo se realizó con base en revisión de literatura. Sirvieron como apoyo los trabajos de Gentry (1993); Forero y Gentry (1989), Rangeletal. (2004), Cronquist (1981), Salero: Diversidad biológica de un (bp-T), volumen XIV de la Flora Brasiliensis (Alfredo Cogniaus), volumen 13 de la Flora de Ecuador (Wurdack), Flora de Panamá, Parte VII, fascículo 3 (A. H. Gleason), volumen VII, segunda parte de la Flora de Venzuela (Wurdack), entre otros; las listas y datos fueron comparados con los ejemplares del Herbario Chocó. Mediante el método de biogeografía estática, la distribución geográfica se determinó ubicando los datos obtenidos en el mapa, en el que se representa cada uno de los géneros en las regiones, cada uno con un punto de color diferente, luego se unen los puntos, mostrando una figura que determina el estado de distribución. Este trabajo se complementó con biogeografía dinámica, es decir, con la realización de un muestreo en tres áreas de 0.4 hectárea (10 transeptos de $5 \times 80$ $\mathrm{m}^{2}$, Tutunendo, Tadó y el Alto Baudó; además, se hicieron colecciones al azar. Se siguió la metodología de Villareal y otros (2004).

Para la identificación y determinación del material colectado, se utilizaron las bases de datos de los herbarios: NY, COL, MO del Humboldt y posteriormente se permaneció durante cinco días en el Herbario Nacional Colombiano, confirmando las identificaciones y determinaciones realizadas previamente, es decir, se revisaron todas las colecciones del Herbario Nacional Colombiano mediante el método comparativo y con la colaboración del especialista en Melastomataceae, profesor Humberto Mendoza y Alvaro Cogollo del JAUM(Herbario del Jardín Botánico de Medellín) y los miembros del grupo de biosistemática que vienen manejando grupos específicos de las Melastomataceae.
Tabla 1

Diversidad de la familia Melastomataceae

\begin{tabular}{|lccc}
\hline Género & NSA* & Chocó & Herbario \\
\hline Aciotis & 30 & 5 & 3 \\
Acisanthera & 17 & 1 & 1 \\
\hline Adelobotris & 25 & 2 & 2 \\
Alloneuron & 7 & 1 & - \\
\hline Arthrostema & 7 & 1 & - \\
Axinaea & 30 & 1 & 1 \\
\hline Bellucia & 18 & 1 & 3 \\
Blakea & 100 & 6 & 3 \\
\hline Catocorine & 1 & 1 & - \\
Centradenia & 7 & 1 & 1 \\
\hline Centronia & - & - & 1 \\
Chaetolepsis & - & - & 1 \\
\hline Clidemia & 120 & 28 & 18 \\
Conostegia & 43 & 12 & 8 \\
\hline Graffenrieda & 44 & 3 & 1 \\
Henriettella & 51 & 2 & 3 \\
\hline Heterotrichun & 15 & 1 & - \\
Leandra & 175 & 5 & 5 \\
\hline Meriania & 50 & 1 & 3 \\
Miconia & 1000 & 55 & 44 \\
\hline Monolena & 10 & 8 & 2 \\
Nepsera & 1 & 1 & 1 \\
\hline Osaea & 91 & 12 & 9 \\
\hline Tessmannianthus & 7 & 1 & 1 \\
\hline Tibouchina & 240 & 2 & 4 \\
Tococa & 54 & 6 & 3 \\
\hline Topobea & 62 & 16 & 8 \\
Triolena & 22 & 5 & 3 \\
\hline & & & \\
\hline
\end{tabular}

* Número de especies en Sur América

\section{RESULTADOS}

En el departamento del Chocó, hay un registro de 181 especies, distribuidas en 26 géneros; de éstas, cuatro géneros y 53 especies no reposan en la colección del herbario de la UTCH (Chocó). Existen además 87 melastomatáceas indeterminadas y 310 sólo determinadas hasta género.

En cuanto a la diversidad el género mayor representado en número de especies fue Miconia con 55, seguido de Clidemia 
con 28 especies y Topobea con 16 especies. Este resultado, es un compendio de la información procesada del herbario, de las publicaciones, las bases de datos del COL.MO, NY y otras floras como Brasil, Panamá, Venezuela, Ecuador, Perú (Tabla $1)$.

A nivel de la distribución, al unir los puntos, se observa la forma ovalada que tiene la familia, lo que indica una expansión y centro de confluencia de géneros como:Miconia, Clidemia, Topobea, Osaea, Blakea, Conostegia, Tibouchina, Triolena, Aciotis, Leandra, Adelobotris, Tococa, Monolena que están más repartidos en las zonas fitogeográficas; su organización en orden descendente muestra el grado de representación de los géneros en las zonas. Además, los géneros Acisanthera, Alloneuron, Arthrostema Meriania, Catocorine, Centradenia, Tessmannianthus, Chaetolepsis, Heterotrichun, Centronia, Henrriettella, tienen una distribución limitada o poco conocida.

Las zonas más ricas en diversidad de géneros fueron la selva pluvial central con 19, la región del San Juan con 17, San José del Palmar con 16, la costa Pacífica con 15. Con menos diversidad se encontraron las tierras altas del Carmen de Atrato con 11, la región del Urabá con 10, la serranía del Darién con ocho y la selva húmeda del norte con siete.

En el muestreo de Tutunendo se encontraron 16 géneros (Aciotis, Adelogotrys, Blakea, Clidemia, Henriettela, Leandra, Miconia, Monolena, Ossaea, Tocota, Topobea y Triolena), faltando determinar tres poblaciones que se encontraron en estado estéril y 13 especies por identificar, para un total de 37 especies y 1,349 individuos. Las especies más abundantes y dominantes son Clidemia killipii (196 individuos), Miconia sp1 (155 individuos), Ossaea spicata (151 individuos) y Tocota guianensis (109 individuos). Las más diversificadas son Miconia sp1 (que presentó en nueve de los 10 transectos, llamado vulgarmente por los campesinos como Pogó, árbol entre 17 y 20 metros de altura) y Miconia nervosa (en siete de 10 transectos); los géneros Aciotis y Grafenrieda son los más restringidos; sólo se encontraron en un transecto de los diez. Los géneros con más especies son Clidemia (con seis especies), Blakea y Miconia (con cinco especies cada uno) (Tabla 2).

En el muestreo realizado en Tadó se encontraron 19 géneros (Aciotis, Adelogotrys, Alloneuron, Acisanthera, Bellucia, Blakea, Clidemia, Conestegia, Grafenrieda, Henriettela, Leandra, Miconia, Monolena, Ossaea, Tessmannianthus, Tocota, Topobea y Triolena), faltando determinar tres poblaciones que se encontraron en estado estéril y 18 especies por identificar, para un total de 59 especies y 1.374 individuos. Las especies más abundantes y dominantes son Ossaea sp (244 indiviudos), Graffenriedasp (193 individuos), Clidemia crenulata(128 individuos), Tocota guianensis (110individuos), Ossaea robustay Ossaea spicata (con 91 individuos cada una)y las especies de mayor diver- sidad fueron Ossaea sp (se presentó en nueve de los 10 transeptos y parece ser un nuevo reporte) y Ossaea spicata y Clidemia crenulata (se reportaron en ocho de los 10 transeptos) y Ossaea robusta(en seis de los 10 transeptos). Los géneros Aciotis, Adelobotrys, Acisanthera son los más restringidos, sólo se encontraron en un transepto de los diez. Además, los siguientes géneros presentan varias especies restringidas a un solo transepto Blakea, Clidemia, Conostegia, Leandra, Miconia, Monolena, Ossaea, Topobea, Triolena. Los géneros con más especies son: Miconia (con 13), Clidemia (con ocho) yBlakea (seis)(Tabla 2).

En el muestreo del Baudó se encontraron 13 géneros: Adelogotrys, Blakea, Clidemia, Graffenrieda, Henriettela, Leandra, Miconia, Monolena, Ossaea, Tessmannianthus, Tocota, Topobea y Triolena), faltando determinar tres poblaciones que se encontraron en estado estéril y 18 especies por identificar, para un total de 37 especies y 906 individuos. Las especies más abundantes y dominantes son Tocota padiciflora (187 individuos), Tococa guianensis (95 individuos) y Triolena spicata (79 individuos).Las especies con mayor diversidad fueron Tococa spadiciflora(se presento en siete de los 10 transeptos) y Tocota guianensis (que se reportó en cinco de los 10 transeptos).

Los géneros Aciotis, Adelobotrys son los más restringidos; sólo se encontraron en un transepto de los diez. Los siguientes géneros presentan varias especies restringidas a uno o dos transeptos Blakea, Clidemia, Conostegia, Leandra, Miconia, Monolena, Ossaea, Tessmannianthus, Topobea, Triolena. Los géneros con más especies son: Miconia (con siete especies), Clidemia (con seis especies), Ossaea y Blakea (cuatro especies cada uno) (Tabla 3). Como muestra esta tabla, el total de géneros colectados fue de 19 y 87 especies en las tres zonas. De igual manera, se muestra comparativamente el número de géneros y especies de cada zona, así: Tutunendo 16/41; Tadó19/55 y Baudó, 13/37.

\section{DISCUSIÓN}

La distribución actual de los individuos de la familia Melastomataceae se correlaciona con el clima, lo que coincide con la afirmación de que el Chocó es el lugar más húmedo del mundo (Forero y Gentry 1989). En estos momentos se encuentra en expansión como lo muestra el Mapa 1, a pesar de que éste es un primer trabajo y que falta adelantar más trabajo de campo y en especial las colecciones al azar.

La riqueza de los géneros Miconia, Leandra, Topobea, Osaea, Conostegia, Tococa, Clidemia, Monolena, Acioitis, Tibouchina, Triolena, Henrrietalla, Graffenrieda, Acisanthera, Meriania, Blakea, Bellusia, Tessmannianthus y Nepsera, de la familia Melastomataceae, es el eje sobresaliente de una de las áreas más húmedas del mundo, la selva pluvial 
Tabla 2

Relación de especies colectadas por cuadrante Tutunendo-Tadó-Baudó

\section{Lista de melastomataceae del Chocó}

Tutunendo Tadó Baudó

1. Allomaieta strigosa (Gleason)Lozano-16243-

2. Aciotis indecora (Bompl) Triana-16187-

Aciotis ornata(Miq) Gleason-16186

Aciotis polystachia (Bompl) Triana-16228-

Aciotis rubricaulis(Martius ex DC)Triana-16226

3. Acisanthera cuadrata Pers. 16227

4. Adelobotrys adscendens (Sw) Triana-16159-16202

Adelobotrys fuscescens Triana-16238-16265

Adelobotrys monticola Gleason-16196

Adelobotrys tessmannii Mgf- 16217

5. Bellucia pentamera Naudin-16221-16255

6. Blakea allotrichia L.Uribe-16261

Blakea alternifolia(Gleason)Gleason-16225

Blakea florigera Gleason-16180

Blakea glabrescens Benth-16275

Blakea jativae Wurdack-16178

Blakea lanuginosa Wurdack-16273-16276

Blakea podagrica Triana-16198-16173-16245

Blakea punctulata (Triana)Wurdack-16244

Blakea setosa-16450

Blakea subconata Berg-16174-16154

7. Clidemia bernardii Wurdack-16263

Clidemia chocoensis Wurdack- 16203

Clidemia crenulata Gleason-16212-16151

Clidemia epiphytica (Triana )Cogn-16156-16234

Clidemia haughtii Wurdack-16192

Clidemia killipii Gleason-16231-16211-16177

Clidemia parasitica O.Berg.-16164

Clidemia septuplinervia Cogn-16257-16224

8. Conostegia eaelestis Standl.-16184

Conostegia cuatrecasii Gleason-16207

Conostegia dentata Triana-16264

Conostegia losiopoda Benth.-16283

Conostegia setosa Triana-16258-16152

9. Graffenrieada anomala Triana-16201

Graffenrieda weddellii Naudin-16175

10. Henriettella fissanthera Gleason-16194

Henriettella goudotiana Naudin-16278-16267

Henriettella laurencei Gleason-16167

Henriettella trachyphylla Triana-16181

11. Leandra granatensis Gleason-16157-16185-16188-16223-16232

Leandra dichotoma(d.Don) Cogn- 16242

12. Miconia albicans (Sw)Triana-16219

Miconia acanthocoryne Wurdack-16190-16240

Miconia acolophoides naudin-16274

Miconia affinis DC-16256-16179

Miconia agreggata Gleason-16270

Miconia argyrophylla DC-16272

Miconia barbinervis (Benth) Triana-16193

Miconia benthamiana Triana-16229

Miconia blanchetiana Naudin-16165

Miconia calathranta Cogn-16213-16155

Miconia calcarata Gleason-16199

Miconia caudata (Bompl)DC-16235

Miconia centrodesma Naudin-16162-16200-

Miconia coronata(Bompl)DC-16214

Miconia curvipetala Cogn ex Gleason-16249

Miconia dorsiloba Gleason-16209

$\mathrm{X}$
$\mathrm{X}$
$\mathrm{X}$
$\mathrm{X}$

$\begin{array}{ll} & X \\ X & X \\ X & X \\ X & X \\ X & X \\ X & X \\ & x\end{array}$

$\mathrm{X}$

$\mathrm{x}$

$x$

$X$
$X$
$X$

$\mathrm{X}$

$\mathrm{X}$

$x$
$X$
$X$
$X$
$X$
$X$
$X$

$X$
$X$
$X$
$X$
$X$
$X$

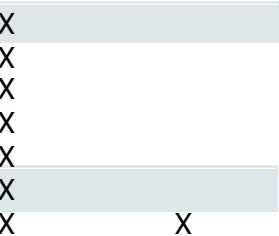

$x$

$x \quad x$

$x \quad x$

$\begin{array}{ll}x \\ x & x \\ x\end{array}$

$\begin{array}{ll}x & x \\ x & x\end{array}$

$x \quad x$

$x \quad x$

$\mathrm{X}$

$x$

$\begin{array}{lll} & & x \\ X & X \\ X & X & \\ X & x & \\ & & \end{array}$

X

$\begin{array}{ll}x & x \\ x & x\end{array}$

$X \quad X \quad X$

$\begin{array}{lll}x & x & x \\ x & x & x\end{array}$

$x$

$\mathrm{X}$

$X$
$X$

$\mathrm{X}$

$\mathrm{X}$

$\mathrm{X}$

$x$

$\begin{array}{ll}x & x \\ x & x\end{array}$

$\begin{array}{ll}x & x \\ x & x\end{array}$

$x$
$x$
$x$

$x$
$x$

$x$

$x$

$\begin{array}{ll}X & \\ X & x \\ X & \end{array}$


Tabla 2

Relación de especies colectadas por cuadrante Tutunendo-Tadó-Baudó (continuación)

\begin{tabular}{|c|c|c|c|}
\hline Lista de melastomataceae del Chocó & Tutunendo & Tadó & Baudó \\
\hline Miconia fulva DC-16150 & & $\mathrm{x}$ & \\
\hline Miconia goniostigma Triana-16220 & & $\mathrm{X}$ & \\
\hline Miconia lonchophylla Naudin-16253 & & $\mathrm{X}$ & \\
\hline Miconia nervosa (Sm)Triana-16222-16160 & $\mathrm{x}$ & $X$ & $\mathrm{x}$ \\
\hline Miconia serrulata Naudin-16191- & $\mathrm{X}$ & & \\
\hline Miconia simplex Triana-16166 & $\mathrm{x}$ & $\mathrm{x}$ & \\
\hline 13. Monolena cordifolia Triana-16271 & & & $\mathrm{X}$ \\
\hline Monolena ovata Cogn-16268 & & & $\mathrm{X}$ \\
\hline Monolena primulaeflora Hook f. $-16170-16247$ & $\mathrm{x}$ & $\mathrm{x}$ & \\
\hline 14. Mouriri grandiflora DC-16246 & & $\mathrm{X}$ & \\
\hline 15. Tessmannianthus calcaratus (Gleason) Wurdack-16193-16208 & $\mathrm{X}$ & $X$ & \\
\hline Tessmannianthus nov sp.16276-16279-16281 & & $X$ & \\
\hline 16.Ossaea bracteata Triana-16172-16215 & $X$ & $\mathrm{X}$ & \\
\hline Ossaea clidemioides Hook f.-16252 & & $\mathrm{X}$ & \\
\hline Ossaea laxivenula Wurdack-16158-16204-16280-16284 & $\mathrm{X}$ & $\mathrm{X}$ & $\mathrm{X}$ \\
\hline Ossaea macrophylla (Benth) Cogn-16161-16189-16197-16251-16282 & $\mathrm{X}$ & $\mathrm{X}$ & $\mathrm{X}$ \\
\hline Ossaea robusta (Triana) Cogn-16239 & $\mathrm{X}$ & $\mathrm{X}$ & $\mathrm{X}$ \\
\hline Ossaea rufibarbis Triana- 16216 & & $\mathrm{X}$ & \\
\hline Ossaea spicata Gleason-16169-16205-16210 & $\mathrm{X}$ & $\mathrm{X}$ & $\mathrm{x}$ \\
\hline 17. Topobea floribunda Gleason-16254 & & $\mathrm{X}$ & \\
\hline Topobea inflata Triana-16171-16183 & $\mathrm{X}$ & & $\mathrm{X}$ \\
\hline Topobea parasitica Aubl.-16163 & $x$ & & \\
\hline 18. Tococa fornicaria Mart var. Gordnei Cogn- 16147 & $X$ & & \\
\hline Tococa quianensis Aubl.-16149-16230-16241-16268 & $\mathrm{X}$ & $\mathrm{x}$ & $\mathrm{x}$ \\
\hline Tococa spadiciflora Triana-16153-16206-16236-16260 & $\hat{x}$ & $\hat{x}$ & $\hat{x}$ \\
\hline 19. Triolena barbeyana Cogn-16259 & & & $\mathrm{X}$ \\
\hline Triolena oblicua(Triana ) Wurdack- & & & $X$ \\
\hline Triolena pedemontana Wurdack-16146 & $\mathrm{x}$ & & \\
\hline Triolena spicata (Triana)L.Wms.-16218-16237-16248 & $\mathrm{x}$ & & \\
\hline Total Géneno/Especie 19/87 Subtotal por estación & $16 / 41$ & $19 / 55$ & $13 / 37$ \\
\hline
\end{tabular}

central, debido a su alta pluviosidad que se evidencia en el número de colecciones y la accesibilidad a estos lugares (Forero y Gentry 1989), lo que se refrenda con los resultados obtenidos en el muestreo de 0.4 hectárea, con 16 géneros de los 26 que se han reportado para el Chocó y de los 19 reportados para Tutunendo en este estudio, enunciado antes; sobresale la dominancia y diversidad del género Miconia y Clidemia. De igual manera las 37 especies obtenidas es un dato interesante al compararlo con las 30 especies que se observan en el estudio de impacto ambiental del Baudó (Animas-Nuquí). Muchas de estas especies tienen una gran utilidad en la farmacopea nativa, para cura de picadura de culebras, diferentes tipos de dolores, golpes, fiebres, gripa, resfriados, etc. En el estudio del Baudó sobresalen de nuevo los géneros Clidemia y Miconia con seis especies cada uno.

La riqueza de los géneros Miconia, Leandra, Topobea, Osaea, Conostegia, Tococa, Clidemia, Monolena, Acioitis, Tibouchina, Triolena, Henrrietalla, Graffenrieda, Acisanthera, Meriania, Blakea, Bellucia, Tessmannianthus y Nepsera, de la familia Melastomataceae, es el eje sobresaliente de una de las áreas más húmedas del mundo, la región del San Juan, por su alta pluviosidad que se evidencia en el número de colecciones y la accesibilidad a estos lugares (Forero y Gentry 1989), lo que se refrenda con los resultados obtenidos en el muestreo de 0.4 hectárea, con 18 géneros de los 26 que se han reportado para el Chocó y de los 17 reportados para el San Juan en este estudio; es decir, sobrepasamos lo enunciado anteriormente. Sobresale la dominancia y diversidad de géneros: Ossaea, Graffenrieda y Tococa que no es el paradigma para el Chocó, pero que nos dice que es su zona de mejor adaptación; de igual manera, las 59 especies que se obtuvieron son un dato interesante al compararlo con las 30 especies que se observaron en el estudio de impacto ambiental del Baudó (ÁnimasNuquí) y las 50 obtenidas en Tutunendo.

Los resultados para el Baudó también son importantes porque se obtienen 13 géneros de los 15 reportados anteriormente por Forero y Gentry (1989) y Rangel (2004); el número de especies coincide con los datos reportados por Rentería (2005), en el estudio de impacto ambiental Ánimas-Nuquí UTCh-INVIAS. A diferencia de Tutunendo donde la especie más dominante fue Clidemia killipii, en el estudio fue Tocota spadiciflora, Tococa guiananesis y Triolena spicata. Un 


\section{Bioetnia Volumen 6 № 2 (julio-diciembre), 2009}

aspecto importante es que el estudio se realizó en un bosque primario, razón por la que el número de géneros y especies es más bajo que en los otros puntos. El género con más especies coincide con Miconia.

La riqueza de los géneros Miconia, Leandra, Topobea, Osaea, Conostegia, Tococa, Clidemia, Monolena, Acioitis, Tibouchina, Triolena, Henrrietalla, Graffenrieda, Acisanthera, Meriania, Blakea, Bellucia, Tessmannianthus y Nepsera, de la familia Melastomataceae, es el eje sobresaliente de una de las áreas más húmedas del mundo, la región del San Juan, por su alta pluviosidad que se evidencia en el número de colecciones y la accesibilidad a estos lugares (Forero y Gentry 1989), lo que se refrenda con los resultados que se obtuvieron en el muestreo de 0.4 hectárea, con 16 géneros de los 26 que se han reportado para el Chocó y de los 19 reportados para el San Juan en este estudio, es decir, se sobrepasa lo enunciado antes. Sobresale la dominancia y diversidad del génerosOssaea, Graffenrieday Tococa que no es el padigma para el Chocó, pero que nos dice que es su zona de mejor adaptación; de igual manera, las 55 especies obtenidas es un dato interesante al compararlo con las 37 especies que se obsevaron en el estudio de impacto ambiental del Baudó (Animas-Nuquí) y las 41 obtenidas en Tutunendo.

Muchas de estas especies tienen una gran utilidad en la farmacopea nativa, para cura de picadas de culebras, diferentes tipos de dolores, golpes, fiebres, gripa, resfriados, etc., como lo demuestra Salas 2007, al realizar una revisión de los trabajos de fotoquímica: «Observando todos los trabajos se nota claramente una constante, referente a los metabolitos secundarios encontrados en las especies de la familia melastomatáceas, y es la gran cantidad de taninos hidrolizables que presentan estas plantas, así como también los flavonoides, sin contar con los compuestos nuevos aislados y las actividades biológicas de estos».Actualmente, él viene realizando un estudio químico de Aciotis polystachya que en el Perú se utiliza para tratar la tuberculosis, enfermedades de dolencias en los riñones (Freire-Fierro 2002), especie que fue colectada en Tadó y de la que se tomó muestra para el análisis químico.

Otro factor que influye es la capacidad de dispersión, que desde el punto de vista ecológico se ve reflejado con los diversos tipos de frutos que la familia brinda, las múltiples y abundantes semillas que contienen los frutos, así como lo apetecido de su néctar, útil para frugívoros y nectarívoros (Cronquist 1981).

Los estudios de Melastomataceae son muy restringidos y en todos los muestreos generales de la vegetación ocupan un lugar importante; analizando los patrones de distribución, se puede ver la importancia de la esquina de América (Chocó), Transecto EIA 0.4 ha (UTCh-INVIAS 2005) con 30 especies. El departamento del Chocó (Forero y Gentry 1989), con 181 especies; Chocó Biogeográfico (Rangel 2004), con 225 especies. Comparado con Barro Colorado Panamá(Croat 1978) que reporta 35 especies, Magdalena medio (Rentería 1980) con 13 especies, San Luis Antioquia (Rentería y Cogollo 1984) con 42 especies, Urabá (Rentería y Brand 1986) con 22 especies y Salero (García et al. 2003) con 4 especies, presentándose un alto volumen de recombinación de especies. Los datos de la estación Tutunendo del alto San Juan y Baudó, muestran que es necesario seguir trabajando en un inventario intensivo en todas las estaciones, para romper los paradigmas tradicionales; es decir, además de los trabajos rápidos como los que se están haciendo hoy, se debe complementar con una mayor dedicación en tiempo a las colecciones al azar. Además, se ratifican la importancia de la realización de estos estudios monográficos de las familias más representativas del departamento, por toda la información ecológica fitogeográfica y sistemática que proporciona.

Al poseer la familia Melastomataceae distribución cosmopolita (viven en todos los países del globo, con condiciones propicias a su desarrollo), facilita o establece una capacidad de dispersión de sus especies que supera la dispersión real y efectiva.

La curva de acumulación de especies de las Melastomataceae (Gráfica 1) muestra al inicio un ascenso rápido y luego una estabilización sin llegar a la saturación, lo que significa que con un esfuerzo superior se lograría un mayor número de especies en las parcelas, como lo muestra la línea de tendencia logarítmica; es decir, el mismo nos está diciendo, que hay que dedicarle más esfuerzo, es decir, más cuadrante en la zona.

Al comparar los resultados de las tres salidas de campo, se observó un total de 87 especies, donde Tadó, a pesar haber presentado un bosque secundario, registró $50 \%$ de los cuadrantes, comparado con Baudó que es un bosque completamente primario y Tutunendo un bosque intervenido, lo que nos demuestra la riqueza de las Melastaomataceae en estos ecosistemas. Un $16 \%$ de las 87 especies están en las tres zonas, $13.7 \%$ se encuentran en Tutunendo y Tadó, 5.7\% comparten entre Tutunendo y Baudó; Tadó y Baudó comparte $4.5 \%$ de las especies, un 13.7 sólo se presenta en exclusivas de Tutunendo, 13.7 son exclusivas del Baudó y 28.7 sólo están presentes en Tadó, es decir que 25 especies sólo se reportan para Tadó, 12 especies sólo para el Baudó y 12 para Tutunendo.

Los géneros con más especies son Miconia Blakea, Clidemia y Ossaea, con 23, 10,8 y 7 especies. Al comparar las 87 especies (que se resumen en 19 géneros) con las bases de datos de la floras de Brasil, Colombia, Ecuador, Panamá, Perú y Venezuela se encuentra que:

- 28 especies son primer registro para el Chocó.

11 especies son primer registro para Colombia.

25 especies endémicas de Colombia.

19 géneros/ 87 especies para el Chocó.

El Chocó comparte 14 especies con Brasil, 42 especies con Ecuador, 40 con Panamá, 19 especies con Perú y 21 con 
Melastoamataceae de tres regiones del Chocó. E Rentería et al.

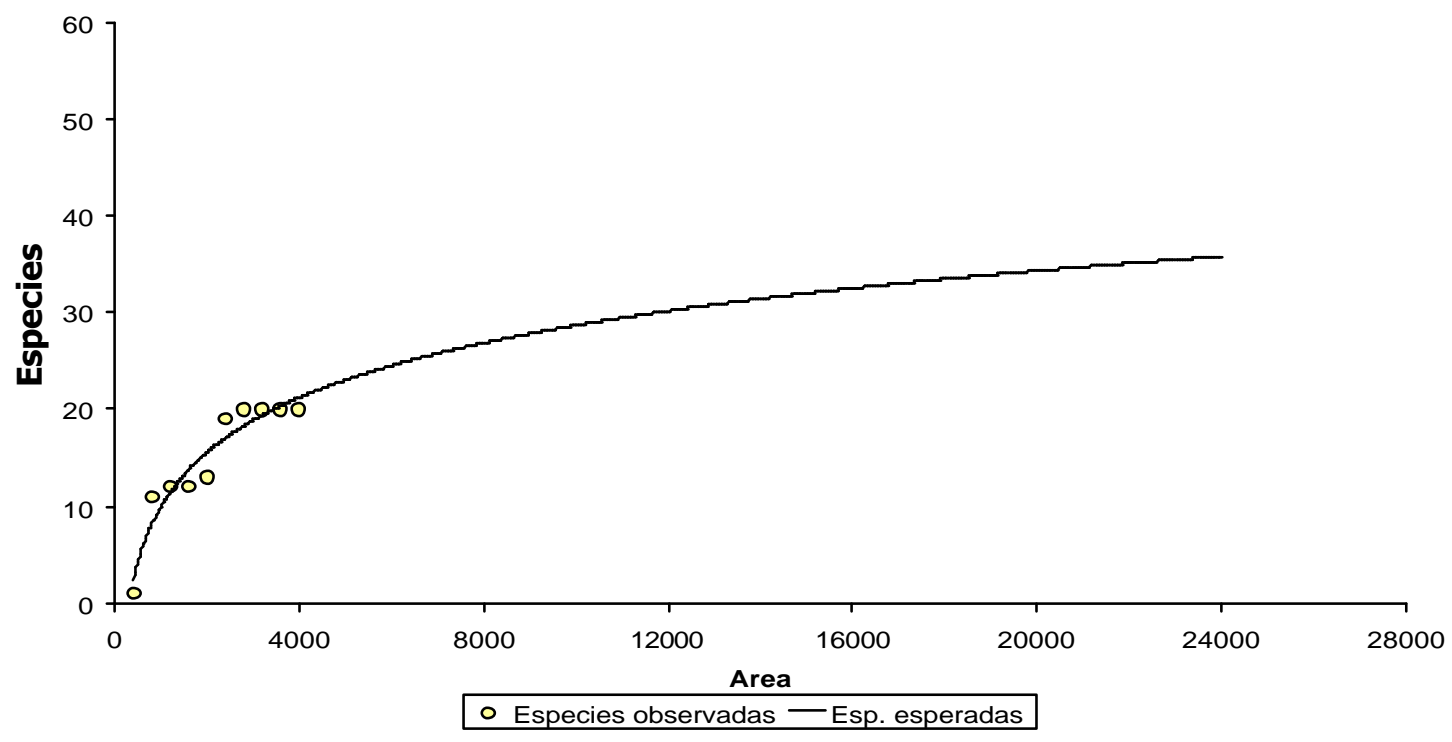

Gráfica 1. Curva de acumulación de especies Melastomataceae.

Venezuela; es decir, que las Melastomataceae del Chocó son más afines con Ecuador, Panamá y Venezuela respectivamente.

\section{CONCLUSIONES Y RECOMENDACIONES}

Frente a la falta de información y sistematización de las pocas expediciones recientes se hace difícil cuantificar el número de colecciones botánicas en el herbario Chocó.

Debido a que la familia Melastomataceae está presente en todos los inventarios florísticos, se hace necesario determinar sistemáticamente y biogeográficamente sus representantes para poder obtener mayor diferenciación de los mismos y disminuir las limitaciones ecológicas, a través de la asociación de los componentes históricos y ecológicos, porque la extensión del área ocupada depende de la latitud ecológica o adaptabilidad de la especie.

Es necesario hacer trabajos de campo para complementar información, enfatizando en las regiones antes mencionadas para actualizar los listados.

Después de terminar el primer estudio de las parcelas en todas las estaciones, se debe completar el estudio con colecciones al azar de ejemplares con flores y/o frutos, lo que dará una mejor información de la familia en la región. De igual manera, se requiere reconfirmar la identificación de los especímenes en el herbario nacional colombiano, que cuenta con mayor información sobre esta familia.

Este estudio permitió corroborar la importancia de realizar este tipo de estudio monográfico, enriquecer la información sobre la familias para realizar múltiples interpretaciones fitogeográficas y descubrir o reconfirmar patrones de distribución, completar la información florística para realizar verdaderas trabajos sistemáticos, conocer mejor la etnobotánica de las especies de esta familia y cómo la comunidad afro e indígena utiliza sus recursos, incluyendo el estudio fotoquímico, tal como se viene trabajando conAciotis polystachya, colectada en Tadó (Salas 2007). Los estudios se deben extender a los demás géneros de gran importancia etnobotánica.

Se obtuvieron 28 especies que son primer registro para el Chocó, 11 especies que son primer registro para Colombia, 25 especies endémicas de Colombia, 19 géneros/ 109 especies y una especie nueva de Tessmannianthus y Henrriettella.

Es posible obtener nuevas especies de otros géneros porque existen muchos materiales estériles, lo que hace necesario hacer seguimiento a sus períodos de floración y fructificación.

\section{AGRADECIMIENTOS}

Sea este el medio para agradecer al IIAP (en especial a sus administradores) por su colaboración para la realización del presente trabajo, a la bióloga Angélica Asprilla, a los miembros de la comunidades, de los consejos comunitarios de Tutunendo, Tadó y Alto Baudó por su acompañamiento y colaboración durante el levantamiento del muestreo; a los trabajadores de la estación del Tutunendo y Alto San Juan y a la Alcaldía del Alto Baudó por faciltar nuestra permanencia en la ciudad, a los demás miembros del grupo de trabajo de palmas, helechos y etnobotánica, con quienes poco a poco vamos integrando un verdadero equipo de trabajo. 


\section{Bioetnia Volumen 6 No 2 (julio-diciembre), 2009}

\section{LITERATURA CITADA}

Cronquist, A. 1981. An integrated system of classification of flowering plants. New York: Columbia University Press: p. 1162.

Cogniaux, A. Melastomaceae. 1891. In: Florabrasilensis. D. C. Monographiae phnerogamarum 7.

Croat, Th. 1978. Flora de barro colorado. California: Stanford University Press. $490 \mathrm{pp}$.

Forero, E., A. Gentry. 1989. Lista anotada de las plantas del departamento del Chocó. Biblioteca J. J. Triana ${ }^{\circ} 19$. Bogotá. DC: Instituto de Ciencias Naturales, Museo de Historia Natural, Universidad Nacional de Colombia.

Cossio, F.G., Mersory, M., Robledo, D., Mosquera, L.J. Palacios, L. 2004 Composición y diversidad florística de los bosques de la cuenca hidrográfica del río Cabí, Quibdó, Chocó. Revista Institucional Universidad Tecnológica del Chocó. 20: 10-8.

Freire-Fierro, A. 2002. Monograph of Aciotis (Melastomataceae). Syst Botany Monograp. 62: 1-99.

Gentry, A. H. 1993. A field guide to the families and genera of woody plants of northwest South America (Colombia, Ecuador y Perú). Washington, DC: Conservation International.

Gleason, H.A. 1958. Melastomataceae en la flora de Panamá. Ann Missouri Botanical Garden. 45 (3): 203-304.

Heywood, V. H. 1978. Flowering plants of the word. Mayflower Book MARTIUS, CAROLUS \& AUGUSTUS

Plan de Desarrollo Departamental. 2004-2007. Desarrollo con equidad «El Anhelo de un Pueblo» Pág. 9-13. Quibdó: Gobernación del Chocó

Quiñones, L. M. 2001. Diversidad de la familia Melastomataceae en la Orinoquía colombiana. Bogotá, DC: Instituto de Ciencias Naturales, Universidad Nacional de Colombia; $126 \mathrm{p}$
Rangel, J. 2004. Colombia diversidad biótica. Tomo 4. En: El Chocó Biogeográfico/Costa Pacífica. Bogotá, DC: Instituto de Ciencias Naturales, Universidad Nacional de Colombia.

Renner, S. S. 1993. Phylogeny and classification of the Melastomataceae and Mernecylaceae. Nord J Bot. 13: 519-40.

Rentería, E. 1980. Estudio botánico de un bosque húmedo tropical localizado en el Magdalena Medio. Bogotá, DC: COLCIENCIAS-Universidad de Antioquia. Informe final. $96 \mathrm{pp}$.

Rentería, E, A. Cogollo. 1984. Estudio florístico y ecológico de la región de San Luis, Antioquia (río Samaná y Claro). Medellín: Fundación La Fe y Jardín Botánico Joaquín Antonio Uribe. Informe final. 35 pp.

Rentería, E, J. Brand. 1986. Estudio botánico y ecológico de Urabá. Bogotá, DC: COLCIENCIAS-Jardín Botánico Joaquín Antonio Uribe. Informe final. $141 \mathrm{pp}$.

Rentería, E. 2005. Estudio de las Melastamatacea del Baudó en estudio de impacto ambiental. Ánimas, Nuquí. Quibdó: Universidad Tecnológia del Chocó, Invías.

Salas, M. H. 2007. Fitoquímica de las Melastomataceae. Trabajo de investigación, Mérida: Universidad de los Andes; 36 pp.

García, F., Y. Ramos, J. Palacios, J.E. Arroyo, A. Mena, M. González. 2003. Interacción planta-animal: caracterización de la folivoría en algunas especies de Melastomataceae. En: Salero: Diversidad biológica de un bosque pluvial tropical $(b p-T)$. Capítulo V, 2 ed. Bogotá, DC: Editorial Guadalupe Ltda; p. 129-36.

Villarreal, H, M. Álvarez, S. Córdoba, F. Escobar, G. Fagua, F. Gast, H. et al. 2004. Manual de métodos para el desarrollo de inventarios de biodiversidad. Bogotá, DC: Instituto de Investigaciones de Recursos Biológicos, Alexander Von Humboldt; 236 pp.

Wurdack, J. J. 1980. Melastomataceae. En: G. Harling, B. Sparre (eds.). Flora de Venezuela. $\mathrm{N}^{\circ} 13$. Stockholm:Universidad Göteborg, Riksmuseum. $\mathrm{p}$. 406 . 